"Capturing the volatility smile: parametric volatility models versus stochastic volatility models"

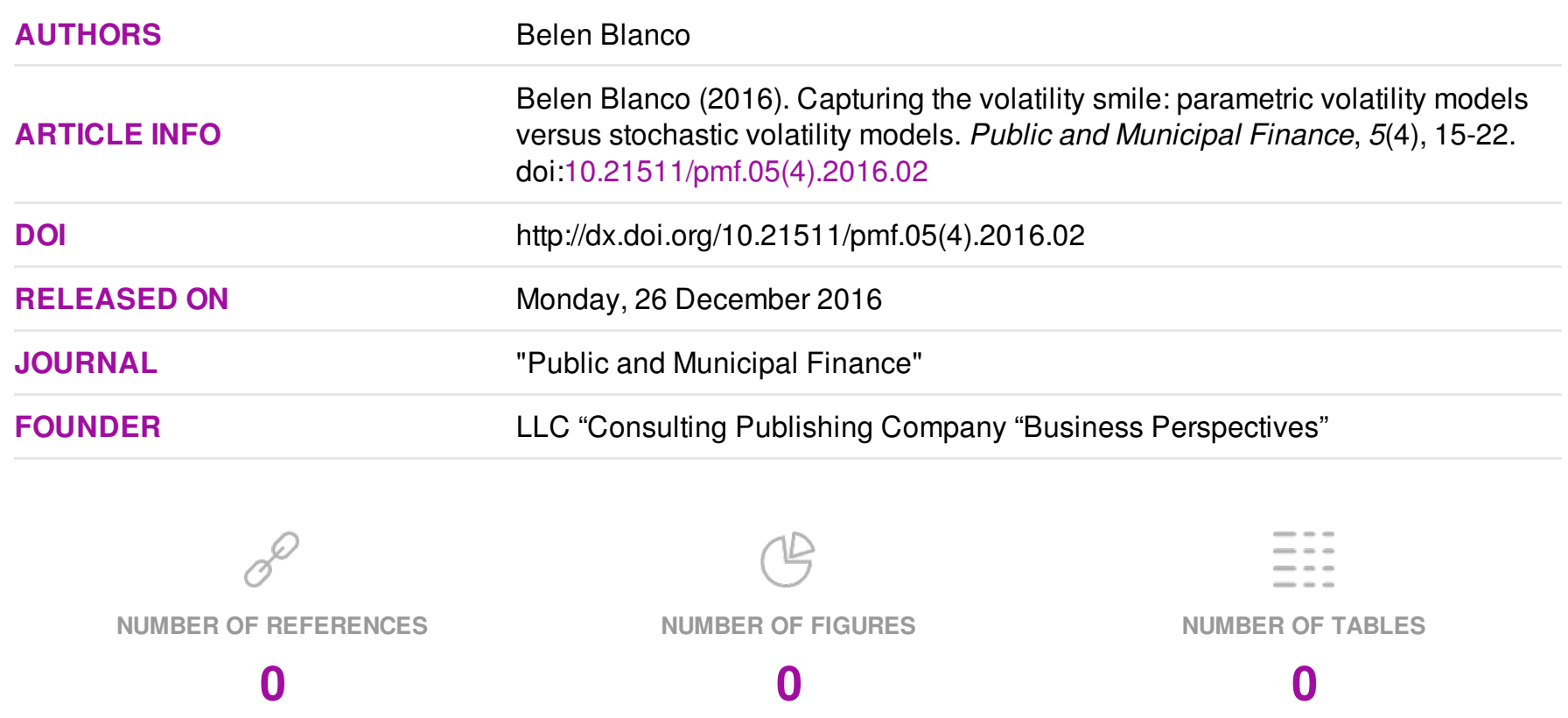

C The author(s) 2023. This publication is an open access article. 
Belen Blanco (Australia)

\title{
Capturing the volatility smile: parametric volatility models versus stochastic volatility models
}

\begin{abstract}
Black-Scholes option pricing model (1973) assumes that all option prices on the same underlying asset with the same expiration date, but different exercise prices should have the same implied volatility. However, instead of a flat implied volatility structure, implied volatility (inverting the Black-Scholes formula) shows a smile shape across strikes and time to maturity. This paper compares parametric volatility models with stochastic volatility models in capturing this volatility smile. Results show empirical evidence in favor of parametric volatility models.
\end{abstract}

Keywords: smile volatility, parametric, stochastic, Black-Scholes.

JEL Classification: C14, C68, G12, G13.

Acknowledgement: I gratefully acknowledge financial support from the Spanish Ministry of Economy and Competition (ECO2013-48328 and ECO2010-19314), and from the Ramón Areces Foundation.

\section{Introduction}

In financial economics, there is a concern about how modelling the volatility, and more specifically, how modelling the volatility in option pricing. There are two main alternatives available for this purpose: the first one is parametric volatility models (as the Dumas et al.'s model (1998)), and the second one is stochastic volatility models (as the Heston's model (1993)).

Under Black-Scholes option pricing model (1973) assumptions, all option prices on the same underlying asset with the same expiration date, but different exercise prices should have the same implied volatility. Empirically, this is not what I observe using traded option prices. Instead of flat implied volatility structure, prior literature finds implied volatility (inverting the Black-Scholes formula) showing a smile shape across strikes and time to maturity (i.e., Cont and Fontseca, 2001; Alerton, 2004). With the aim to fit the implied volatilities, Derman \& Kani (1994), Dupire (1994) and Rubinstein (1994), among others, develop a volatility function that fits the observed cross-section of option prices. A more recent stream of literature uses parametric models to fit the implied volatilities (Ncube, 1996; Dumas et al., 1998; Peña et al., 1999).

In contrast, other stream of literature uses stochastic volatility models to fit the volatility surface. The paper of Hull and White (1987) was the first systematic approach in option pricing literature to recognize nonconstant volatility. They show that the price of a European option is the Black-Scholes price integrated over the probability distribution of the average variance during the life of the option. Later, Heston (1993) shows that a closed-form solution for a European call can be derived as an integral of the future security price density, calculated by an

(C) Belen Blanco, 2016.

Belen Blanco, Dr., The University of Adelaide, Australia. inverse Fourier transform, what is potentially more precise than the approximation suggested by Hull and White (1987). More recently, Bakshi et al. (1997), Ball and Roma (1994), Bates (1996), among others, analyze if stochastic volatility or random jumps resolve the anomalies in the Black-Scholes model. They find that stochastic volatility models seem to behave slightly better than jumps.

The main objective in this paper is to compare the performance of stochastic volatility models with that of parametric volatility models. To this aim, I use data of transaction prices for future call ${ }^{1}$ options on the Spanish IBEX-35 stock exchange index. I employ a database transacted on $6^{\text {th }}$ of November 2,015. To estimate stochastic volatility models, I use the Heston's model (1993); on the other hand, to estimate parametric volatility models, I use the Dumas et al.'s model (1998), but with small variations in the variables.

The results obtained show empirical evidence in favor of parametric volatility models, respect to the stochastic volatility models. In particular, I find that the parametric volatility models fit the data better than the stochastic volatility models, because the last ones tend to overprice out of the money calls.

This paper is organized as follows: the next section contains a description of how obtaining the implied volatility. In section 2 , I present the parametric volatility models, which are based on some adaptations of the parametric models from Dumas et al. (1998), to estimate the surface across moneyness. In section 3, I describe the Heston's model (1993) as an approach to stochastic volatility models. The data are

\footnotetext{
${ }^{1}$ In principle, call and put options should yield the same implied volatility, based on the Call Put parity theorem. Some argue (Ncube, 1996) that since the put option is a natural hedging instrument, investors may be willing to pay more for it and, therefore, its implied volatility would be higher than the call counterpart. However, I will not take into account this possible bias in my model, since I am interested in obtaining a more generic solution, from which both call and put options can be priced.
} 
described in section 4. The estimation results of the volatility surfaces for both models are discussed in section 5, I conclude in last section.

\section{Obtaining the implied volatility with the Newton-Raphson algorithm}

The Black-Scholes European call option formula is the following one:

$$
C=S_{0} e^{-q(T-t)} N\left(d_{1}\right)-K e^{-r(T-t)} N\left(d_{2}\right),
$$

where $S_{0}$ is the underlying asset (in this case, the future contract of IBEX-35 index), $q$ is the expected dividends paid over the option's life, $X$ is the option's strike price, $(T-t)$ is the time to expiration, $r$ is the risk-free interest rate,

$$
\begin{aligned}
& d_{1}=\frac{\ln \left(S_{0} / K\right)+\left(r-q+\sigma^{2} / 2\right)(T-t)}{\sigma \sqrt{T-t}}, \\
& d_{2}=d_{1}-\sigma \sqrt{T-t},
\end{aligned}
$$

Model 0: $\sigma(M N, T)=\beta_{0}+\varepsilon$,

Model 1: $\sigma(M N, T)=\beta_{0}+\beta_{1} \log (M N)+\beta_{2} \log (M N)^{2}+\varepsilon$,

Model 2: $\sigma(M N, T)=\beta_{0}+\beta_{1} \log (M N)+\beta_{2} \log (M N)^{2}+\beta_{3} T+\beta_{4} T \log (M N)+\varepsilon$.

Model 0 is the volatility function representing the constant volatility as in the Black-Scholes model. Model 1 captures the quadratic volatility smile across moneyness, and model 2 captures extends model 1 by capturing the variation across time, and a combined effect of time and moneyness.

The moneyness in this work is defined as $M N=$ $K / F$. If $K / F>1$, the call option is out-the-money (OTM), and when $K / F<1$, the call option is in-themoney (ITM). When $K / F \approx 1$, I can say that the call option is at-the-money (ATM).

$\beta_{0}$ is the constant of the regression. $\beta_{1}$ coefficient captures the dislocation of the origin of the parabola with respect to the ATM options, and $\beta_{2}$ coefficient controls the size of the smile. $\beta_{3}$ and $\beta_{4}$ capture the term structure of the implied volatility.

$\beta$ vector is estimated with nonlinear least-squares function as follows:

$$
\min \operatorname{SSE}(\beta)=\sum_{i=1}^{m}\left(y_{i}-\operatorname{prd}_{i}(w)\right)^{2}
$$

where $\operatorname{prd}_{i}(w)$ is the function that implements each specific model of the volatility surface equation.

Later, I measure how successful the fit of the model is in explaining the variation of the data with the $R^{2}$ statistic. For the nonlinear least squares estimation, is defined as the square of the correlation between the observations (Greene, 2000): $\sigma$ is the volatility rate, and $N(d)$ is the cumulative unit normal density function with upper integral limit $d$. The implied Black-Scholes volatility can be found individually from traded option prices:

$\frac{\partial B S}{\partial \sigma}>0$.

The Newton-Raphson algorithm provides a numerical way to invert the Black-Scholes formula in order to recover $\sigma$ from the market prices of the call option $\mathrm{C}$ (or Put option P)

$$
f(\sigma)=B S(\sigma)-C=0 .
$$

\section{The parametric volatility models}

To estimate parametric volatility models, I use an adaptation of the parametric models proposed by Dumas et al. (1998). In particular, I run the following three models, as a function of moneyness and time:

$$
R^{2}=1-\frac{\operatorname{SSE}(\beta)}{\sum_{i=1}^{m}\left(y_{i}-\bar{y}\right)^{2}} .
$$

From this formula, I can calculate the adjusted $\mathrm{R}^{2}$ statistic:

$\bar{R}^{2}=1-\left(\frac{n-1}{n-p}\right)\left(1-R^{2}\right)$,

where $p$ is the number of variables.

I will choose the model with higher $\mathrm{R}^{2}$ to compare it to stochastic volatility model.

\section{The stochastic volatility models}

To estimate stochastic volatility models, I use the Heston's (1993) model. Heston (1993) proposed the following model:

$$
\begin{aligned}
& d S_{t}=\mu S_{t} d t+\sqrt{V_{t}} S_{t} d W_{t}^{1}, \\
& d V_{t}=\kappa\left(\theta-V_{t}\right) d t+\sigma \sqrt{V_{t}} d W_{t}^{2}, \\
& d W_{t}^{1} d W_{t}^{2}=\rho d t,
\end{aligned}
$$

where $\left\{S_{t}\right\}_{t \geq 0}$ and $\left\{V_{t}\right\}_{t \geq 0}$ are the price and volatility processes, respectively, and $\left\{W_{t}^{1}\right\}_{t \geq 0},\left\{W_{t}^{2}\right\}_{t \geq 0}$ are correlated Brownian motion processes (with correlation parameter $\rho$ ). $\mu$ is the instantaneous expected rate of return of the underlying asset. $\left\{V_{t}\right\}_{t \geq 0}$ 
is the instantaneous stochastic variance, $\theta$ is the long term mean of the variance, and rate at which the variance converges to this mean is $\kappa . \sigma$ is referred to as the volatility of the variance process. All the parameters $\mu, \kappa, \sigma, \theta$ and $\rho$ are time and state homogenous.

If $\rho>0$, then, volatility will increase as the asset price/return increases. Conversely, if $\rho<0$, then, volatility will increase, when the asset price/return decreases. $\rho$, therefore, affects the skewness of the distribution. When $\sigma$ is 0 , the volatility is deterministic and, hence, the log-returns will be normally distributed. Higher $\sigma$ makes the skew/smile more prominent and means that the volatility is more volatile. $\kappa$, the mean reversion parameter, can be interpreted as representing the degree of volatility

$d \tilde{W}_{t}^{1}=d W_{t}^{1}+v_{t} d t$,

$d \tilde{W}_{t}^{2}=d W_{t}^{2}+\Lambda(S, V, t) d t$,

$\frac{d Q}{d P}=\exp \left\{-\frac{1}{2} \int_{0}^{t} v_{s}^{2}+\Lambda(S, V, s) d s-\int_{0}^{t} v_{s} d W_{s}^{1}-\int_{0}^{t} \Lambda(S, V, t) d W_{t}^{2}\right\}$,

$v_{t}=\frac{\mu-r}{\sqrt{V_{t}}}$,

where $P$ is the real world measure and $\left\{\tilde{W}_{t}^{1}\right\}_{t \geq 0}$ and $\left\{\tilde{W}_{t}^{2}\right\}_{t \geq 0}$ are $Q$-Brownian Motions. $\Lambda(S, V, t)$ is called the market price of volatility risk. Under measure $Q$, (9) becomes

$$
\begin{aligned}
& d S_{t}=r S_{t} d t+\sqrt{V_{t}} S_{t} d \tilde{W}_{t}^{1}, \\
& d V_{t}=\kappa *\left(\theta *-V_{t}\right) d t+\sigma \sqrt{V_{t}} d \tilde{W}_{t}^{2}, \\
& d \tilde{W}_{t}^{1} d \tilde{W}_{t}^{2}=\rho d t,
\end{aligned}
$$

where,

$$
\begin{aligned}
& \kappa^{*}=\kappa+\lambda, \\
& \theta^{*}=\frac{\kappa \theta}{\kappa+\lambda} .
\end{aligned}
$$

Under the risk-neutral measure, $\lambda$ has effectively been eliminated. In a complete market, every as clustering. This is something that is observed in the market, large price variations are more likely to be followed by large price variations.

3.1. Risk neutral approach. Risk neutral valuation is the pricing of a contingent claim in an equivalent martingale measure (EMM). The price is evaluated as the expected discounted payoff of the contingent claim, under the EMM Q. So,

$$
\text { Option Value }=E_{t}^{Q}\left[e^{(T-t)} H(T)\right] \text {, }
$$

where $H(T)$ is the payoff of the option at time $T$ and $r$ is the risk free rate of interest over $[t, T]$.

Moving from a real world measure to an EMM is achieved by Girsavov's theorem. In particular,

$$
\begin{aligned}
& P_{j}\left(x, V_{t}, T, K\right)=\frac{1}{2}+\frac{1}{\pi} \int_{0}^{\infty} \operatorname{Re}\left(\frac{e^{-i \phi \ln (k)} f_{j}\left(x, V_{t}, T, \phi\right)}{i \phi}\right) d \phi ; \\
& x=\ln \left(S_{t}\right), \quad f_{j}\left(x, V_{t}, T, \phi\right)=\exp \left\{C(T-t, \phi)+D(T-t, \phi) V_{t}+i \phi x\right\}, \\
& C(T-t, \phi)=r \phi i r+\frac{a}{\sigma^{2}}\left[\left(b_{j}-\rho \sigma \phi i\right) \tau-2 \ln \left(\frac{1-g e^{d r}}{1-g}\right)\right], \\
& D(T-t, \phi)=\frac{b_{j}-\rho \sigma \phi i+d}{b_{j}-\rho \sigma \phi i-d}\left(\frac{1-e^{d r}}{1-g e^{d r}}\right), \\
& g=\frac{b_{j}-\rho \sigma \phi i+d}{b_{j}-\rho \sigma \phi i-d}, \quad d=\sqrt{\left(\rho \sigma \phi i-b_{j}\right)^{2}-\sigma^{2}\left(2 u_{j} \phi i-\phi^{2}\right)},
\end{aligned}
$$


for $\mathrm{j}=1,2$, where

$u_{1}=\frac{1}{2}, \quad u_{2}=-\frac{1}{2}, \quad a=\kappa \theta, \quad b_{1}=\kappa+\lambda-\rho \sigma$,

$b_{2}=\kappa+\lambda$.

In this formula, what is really difficult to solve is the equation (15). This integral cannot be evaluated exactly, but it can be approximated with reasonable accuracy by using some numerical integration techniques.

Under EMM, Q some parameters simplification takes place:

$$
a=\kappa^{*} \theta^{*}, b_{1}=\kappa^{*}-\rho \sigma, b_{2}=\kappa^{*} \text {. }
$$

The parameter $\lambda$ has been eliminated.

A method to evaluate formulas in the form of (14) has been proposed by Carr \& Madan (1999). I use this method ${ }^{1}$ in order to solve the problem (see Carr \& Madan for more details about this method).

To estimate the parameter vector, I follow the same process as for $\beta$ vector (equation (6)). I examine the goodness of fit statistics as in (7) and (8).

\section{The data and variable measurement}

The database is comprised of all call options on the future of IBEX-35 index traded daily on MEFF during $6^{\text {th }}$ of November 2.015 for different expiration dates. The set of observations includes only calls which have acceptable level of moneyness ${ }^{2}$. Moreover, I eliminate from the sample all call prices that violate the wellknown arbitrage bounds.
These exclusionary criteria yield a final sample of 113 observations. Then, I estimate the implied volatility for each of the 113 options. I take as the underlying asset the average of the bid and ask price quotation given for each futures contract associated with each call option. I am allowed to use futures prices given that the expiration day of the futures and option contracts systematically coincides during the expiration date cycle. Moreover, note that dividends are already taken into account by the futures price. To proxy for riskless interest rate, I use the rate which gives MEFF to use in order to price derivatives, so I take a rate of $3.03 \%$, and is fixed for all expiration dates.

I define moneyness as the ratio between the exercise price and the average of the futures price relative to each average implied volatility as previously obtained.

\section{Empirical analysis}

5.1. Parametric volatility model. Each of the three models was estimated for the data set, using the estimator described in the section 3 . Table 1 below shows the estimated constant parameter for model 0 , and Figure 1 below shows the implied volatility surface for $6^{\text {th }}$ November 2.015. As expected, the volatility surface is a simple flat surface. Nevertheless, this benchmark model will be used for comparison purposes.

Table 1. Estimated parameters for model 0, RMSE and $\mathrm{R}^{2}$ statistic

\begin{tabular}{|c|c|c|}
\hline$\beta_{0}$ & RMSE & adjR $R^{2}$ \\
\hline 0.1208 & 0.0420 & 0 \\
\hline
\end{tabular}

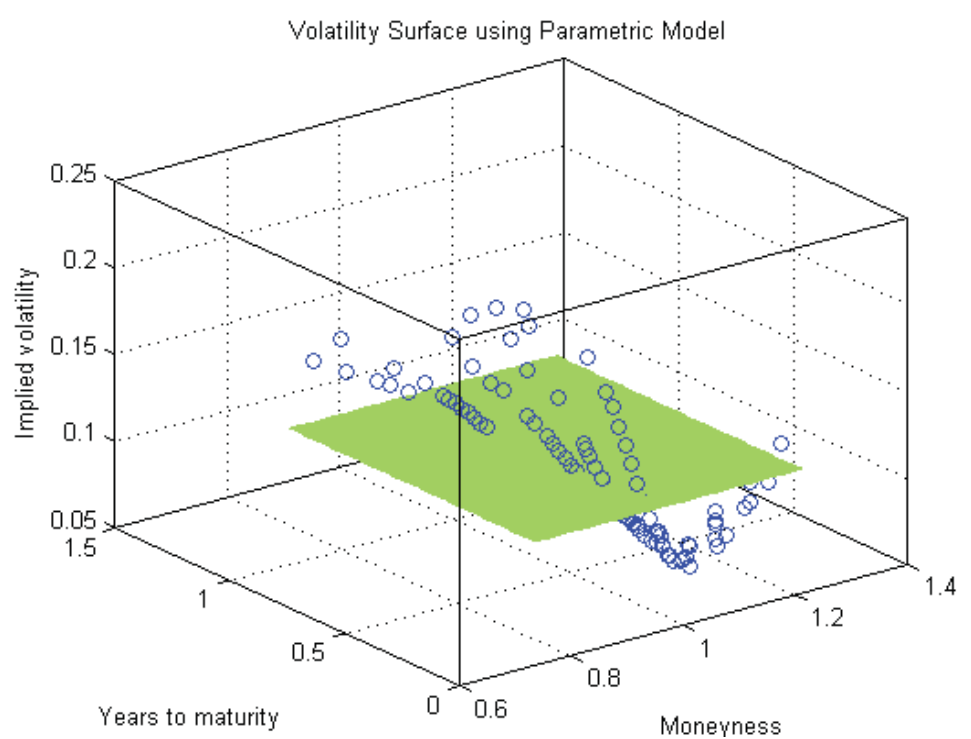

Fig. 1. Estimated implied volatility surface for model 0 . The blue circles are the observed volatilities

The estimation results for model 1 can be found in Table 2 below. When comparing the RMSE values of model 0 with model 1 , I can see that model 1 , with an

\footnotetext{
${ }^{1}$ This method use Fast Fourier Transform in order to solve the problem. It is known as FFT.

${ }^{2}$ Moneyness is going to be between 0.7 and 1.3 in order to avoid problems with extreme values of implied volatility.
}

RMSE of $1.7 \%$, is a considerable improvement over model 0 , which had a RMSE of $4.2 \%$. If I look at the goodness of fit of model 1 with the $\mathrm{R}^{2}$ statistic, the model has a value of 0.8292 , that is, it is able to explain $82.92 \%$ of the variance on the implied volatility. The benchmark model has an $\mathrm{R}^{2}$ of zero, since it is a constant function. 


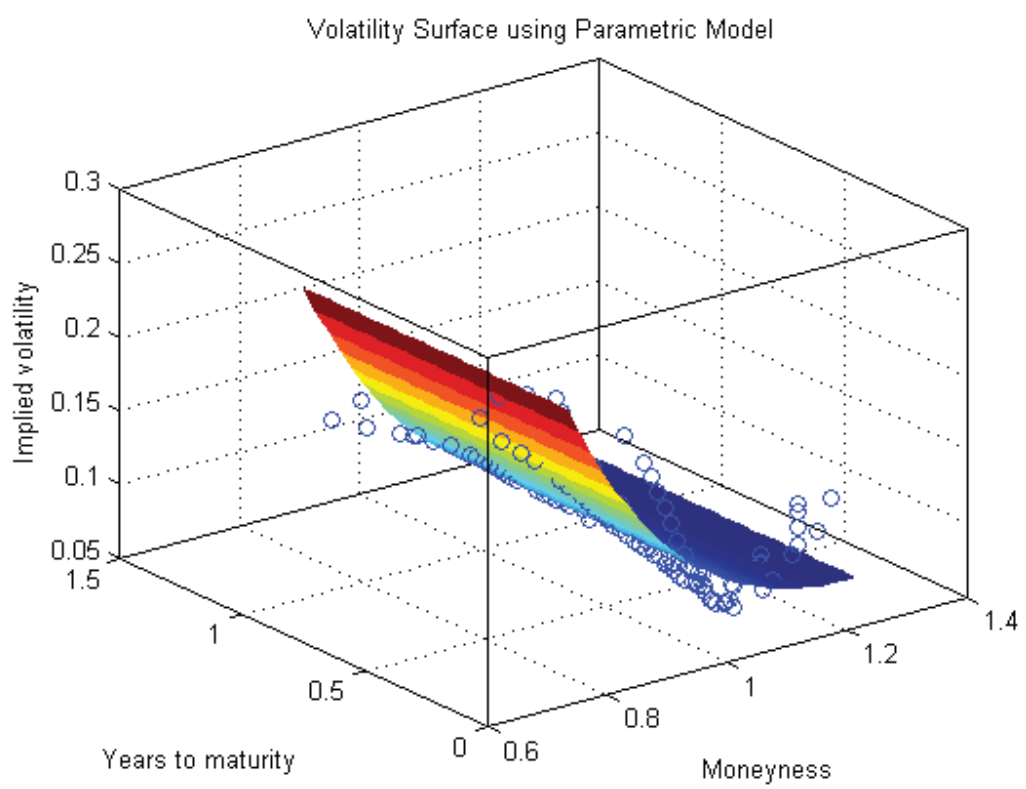

Fig. 2. Estimated implied volatility surface for model 1. The blue circles are the observed volatilities

Table 2. Estimated parameters for model 1, RMSE and $R^{2}$ statistic

\begin{tabular}{|c|c|c|c|c|}
\hline$\beta_{0}$ & $\beta_{1}$ & $\beta_{2}$ & RMSE & adjR \\
\hline 0.1053 & -0.2499 & 0.7187 & 0.0170 & 0.8292 \\
\hline
\end{tabular}

And finally, the estimated parameters for model 2, and a sample implied volatility surface, can be found in Table 3 and Figure 3 below. The RMSE for model 2 is $1.41 \%$, with a $\mathrm{R}^{2}$ statistic of $88.30 \%$. This model, with two additional parameters to capture the term structure dependency, results on a $0.29 \%$ reduction of the RMSE with respect to model 1 , and on a $5.38 \%$ improvement on explaining the variance of the volatility.

Table 3. Estimated parameters for Model 2, RMSE and $\mathrm{R}^{2}$ statistic

\begin{tabular}{|c|c|c|c|c|c|c|}
\hline$\beta_{0}$ & $\beta_{1}$ & $\beta_{2}$ & $\beta_{3}$ & $\beta_{4}$ & RMSE & adjR $R^{2}$ \\
\hline 0.1025 & -0.2698 & 1.2764 & 0.0105 & -1.6707 & 0.0141 & 0.8830 \\
\hline
\end{tabular}

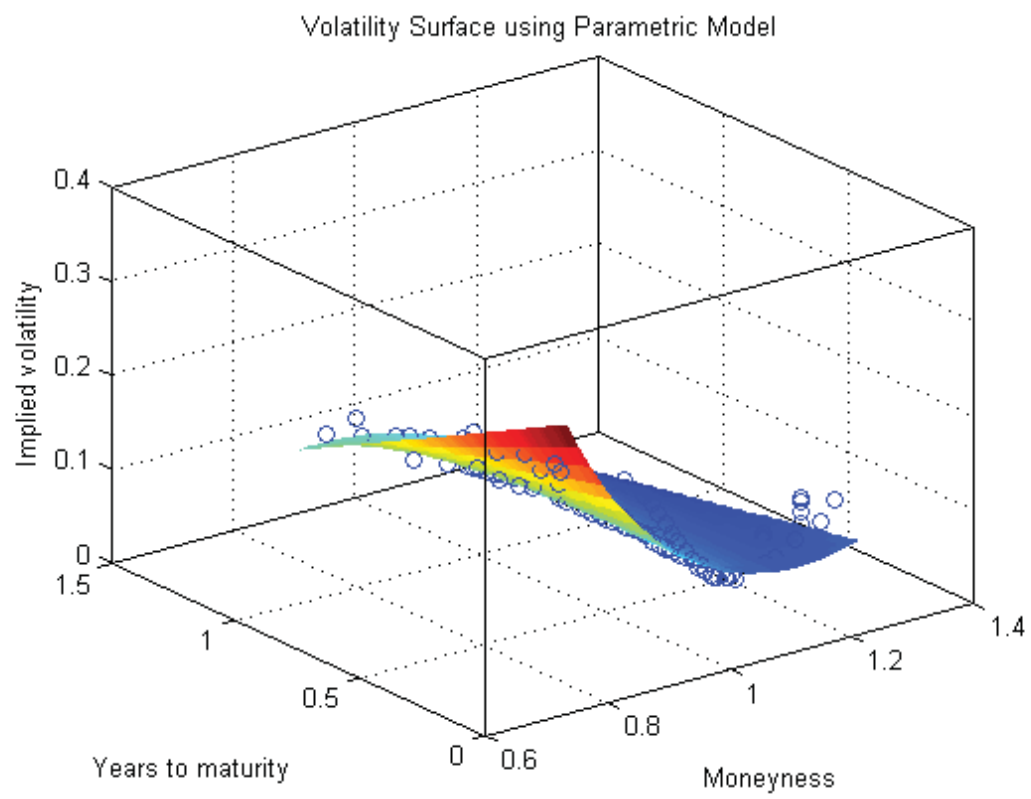

Fig. 3. Estimated implied volatility surface for model 2. The blue circles are the observed volatilities

The best model estimating the volatility surface is the model 2, so I choose this model in or der to compare it with the stochastic volatility model.

5.2. Stochastic volatility model. This model was estimated for the data set, using the risk neutral valuation described in section 4 . Table 4 below shows the estimated constant parameter for Heston's model, and Figure 4 below shows the implied volatility surface for $6^{\text {th }}$ Novem-ber 2015 . 
Table 4. Estimated parameters for Heston's model and $R^{2}$ statistic

\begin{tabular}{|c|c|c|c|c|c|}
\hline$r$ & $\theta^{*}$ & $K^{*}$ & $\sigma$ & $\rho$ & $\operatorname{adj}^{2}$ \\
\hline 0.0127 & 0.2495 & 19.6490 & 1.1233 & -0.5530 & 0.5188 \\
\hline
\end{tabular}

If I look at the goodness of fit of the Heston's model $\quad 0.5188$, that is, it is able to explain $51.88 \%$ of the with the $\mathrm{R}^{2}$ statistic, the model has a value of variance on the implied volatility.

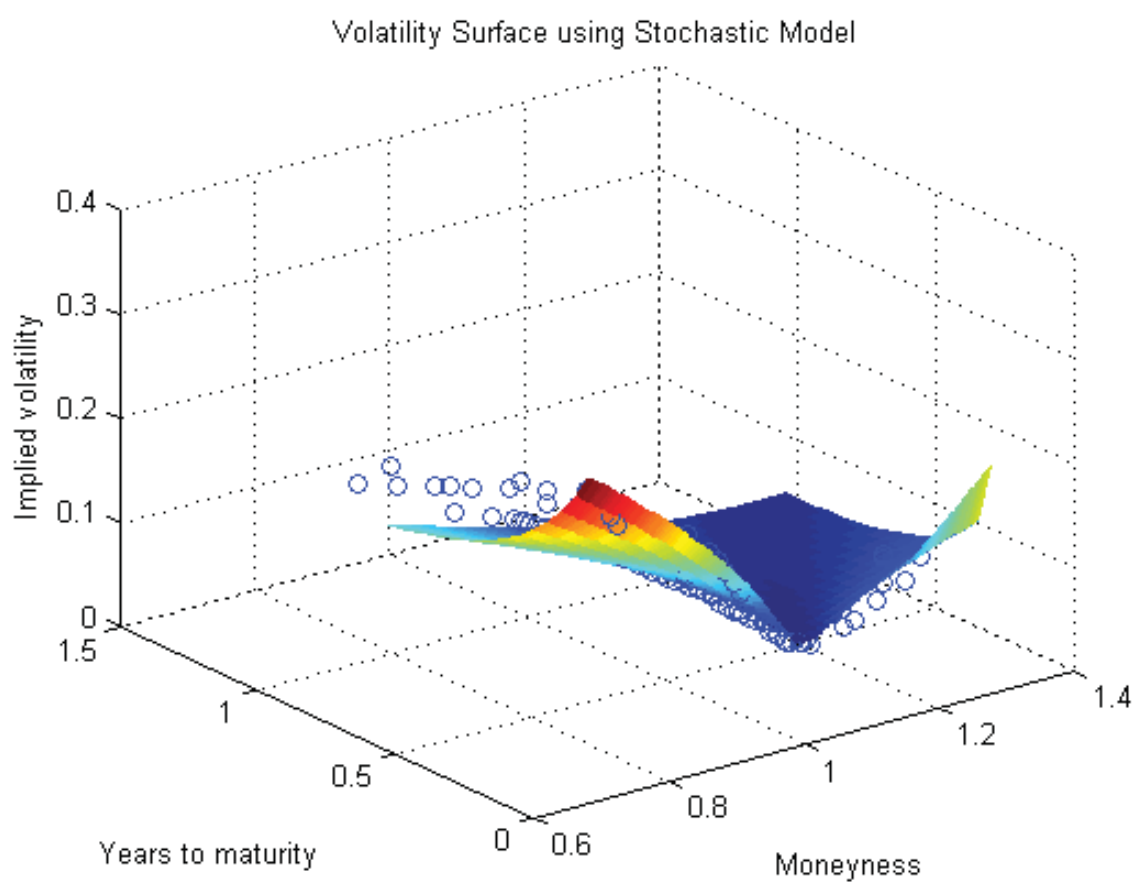

Fig. 4. Estimated implied volatility surface for Heston's model. The blue circles are the observed volatilities

I can see that parametric volatility model (model 2) is able to explain more variance on the implied volatility. The results obtained show empirical evidence in favor of parametric volatility models, respect to the stochastic volatility models. In par- ticular, I find that the parametric volatility models fit the data better than the stochastic volatility models, because the last one tends to overprice out of the money calls. I can see this fact if I rotate the figures.

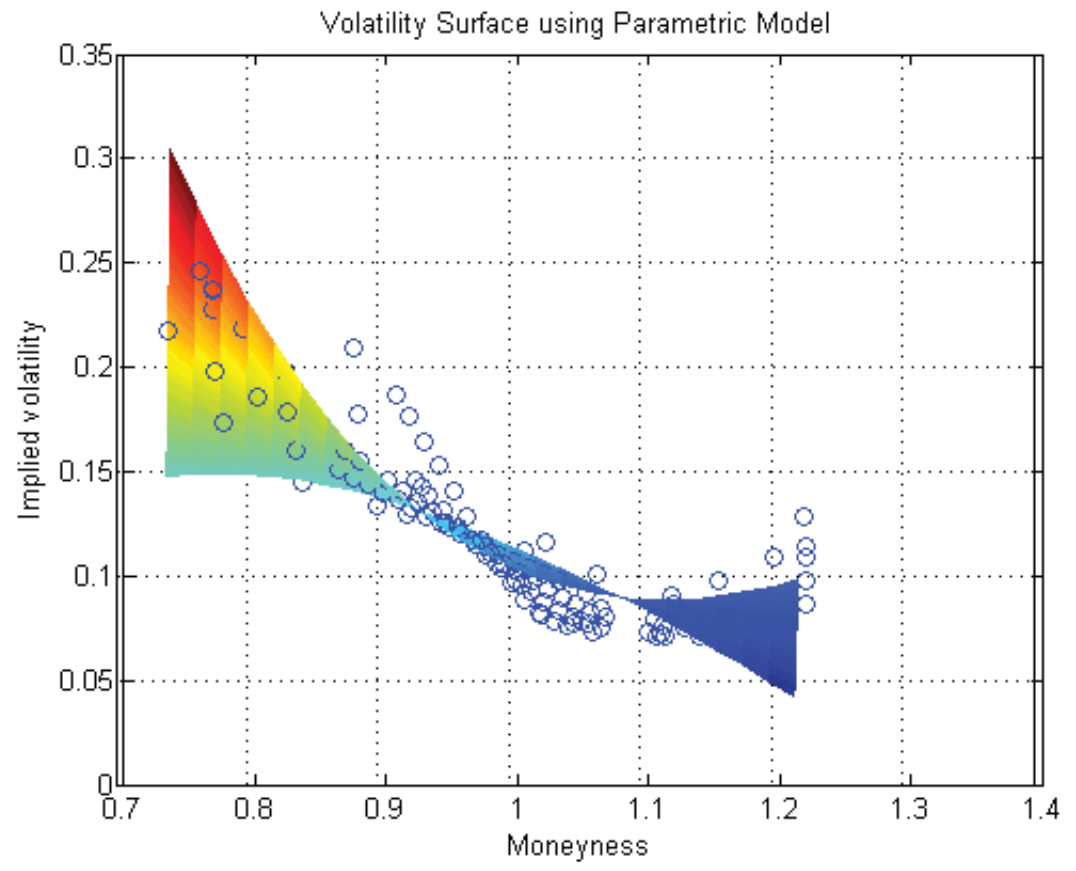

Fig. 5. Estimated implied volatility surface (2D) for model 2. The blue circles are the observed volatilities 


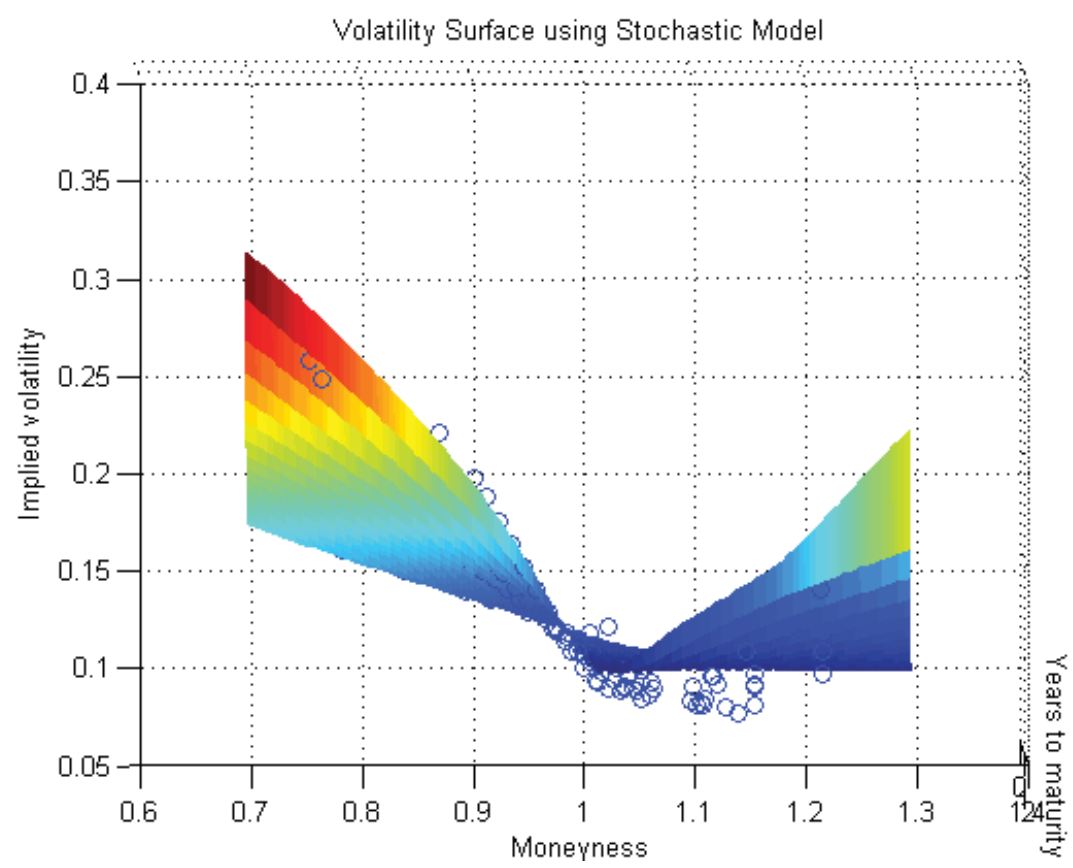

Fig. 6. Estimated implied volatility surface (2D) for Heston's model. The blue circles are the observed volatilities

If I focus only in OTM options in the Heston's model, I can see that the estimate volatility is always higher than the real volatility, and this implies directly that the call price should be higher than in the case of Black-Scholes option pricing model, because volatility and option price are directly and positively associated.

\section{Conclusion}

In financial economics, there is a concern about how modelling the volatility in option pricing. There are two main alternatives available for this purpose: the first one is a parametric volatility model (as the Dumas et al.'s model (1998)), and the second one is a stochastic volatility model (as the Heston's model (1993)).

My main objective in this paper is to compare the performance of the stochastic volatility model with that of parametric volatility model. For this purpose, I employ a database of transaction prices for future options on the Spanish IBEX-35 stock exchange index transacted in $6^{\text {th }}$ of November 2015. To estimate stochastic volatility models, I use the Heston's model (1993); on the other hand, to estimate parametric volatility models I use the Dumas et al.'s model (1998), but with small variations in the variables.

The model that best fits the data is model 2, which captures both the smile across moneyness, as well as the term structure across time to expiration. So the results obtained show empirical evidence in favor of parametric volatility models, respect to the stochastic volatility models. In particular, I find that parametric volatility models fit the data better than stochastic volatility models, because the last ones tends to overprice out of the money calls. This is an important result, as stochastic volatility models are widely used in the financial community as a refinement of the BlackScholes model.

\section{References}

1. Alerton, A. (2004). Modelling the implied volatility surface: an empirical study for FTSE options, Working Paper.

2. Bakshi, G., Cao, C. \& Chen, Z. (1997). Empirical performance of alternative option pricing models, Journal of Finance, 52, pp. 2003-2049.

3. Ball, C., Roma, A. (1994). Stochastic volatility option pricing, Journal of Financial and Quantitative Analysis, 29 , pp. 589-607.

4. Bates, D. (1996). Jumps and stochastic volatility: Exchange rate processes implicit in Deutsche mark options, Review of Financial Studies, 9, pp. 69-107.

5. Black, F., M. Scholes (1973). The Pricing of Options and Corporate Liabilities, Journal of Political Economy, 81, pp. 637-654.

6. Carr, P. \& Madan, D. B. (1999). Option evaluation the using fast fourier transform, Journal of Computational Finance, 2 (4), pp. 61-73.

7. Cont, R. and Fonseca, J.D. (2002). Dynamics of implied volatility surfaces, Quantitative Finance, 2, pp. 45-60.

8. Derman, E. and Kani, I. (1994). Riding on a smile, Risk, 7, pp. 32-39.

9. Dumas, B., Fleming, J. and Whaley, R.E. (1998). Implied Volatility Functions: Empirical Tests, The Journal of Finance, 6, pp. 2059-2106. 
10. Dupire, B. (1994). Pricing with a smile, Risk, 7, pp. 18-20.

11. Greene, W. (2000). Econometric Analysis. Fourth Edition. Prentice-Hall, Inc.

12. Heston, S.L. (1993). A closed-form solution for options with stochastic volatility with applications to bonds and currency options, The Review of Financial Studies, 6 (2), pp. 327-343.

13. Hull, J. and White, A. (1987). The pricing of options on assets with stochastic volatilities, Journal of Finance, 42, pp. 281-300.

14. Ncube, M. (1996). Modelling implied volatility with OLS and panel data models, Journal of Banking and Finance, 20, pp. 71-84.

15. Peña, I., Serna, G, and G. Rubio (1999). Why do we smile? On the determinants of the implied volatility function, Journal of Banking and Finance, 23, pp. 1151-1179.

16. Rubinstein, M. (1994). Implied binomial trees, Journal of finance, 49, pp. 771-818. 